\title{
ORIBÁTIDOS (ACARI: ORIBATIDA): INDICADORES DE IMPACTO ANTRÓPICO EN PARQUES URBANOS DE LA PLATA (ARGENTINA)
}

\author{
Cecilia ACCATTOLI \& Ana SALAZAR MARTÍNEZ \\ División Entomología, Museo de La Plata. Universidad Nacional de La Plata. \\ $<$ ceaccattoli@fcnym.unlp.edu.ar>
}

Accattoli, C. \& Salazar Martínez, A. 2012. Oribátidos (Acari: Oribatida): indicadores de impacto antrópico en parques urbanos de La Plata (Argentina). Acta Zoológica Mexicana (n. s.), 28(3): $550-565$.

RESUMEN. Fueron muestreadas siete áreas verdes urbanas en la ciudad de La Plata con el fin de poner a prueba un índice de intervención antrópica $\left(\mathrm{I}_{\mathrm{A}}\right)$ para suelos urbanos, que surgió de una investigación realizada en el Paseo del Bosque (La Plata, Argentina) y que emplea oribátidos como bioindicadores. $\mathrm{I}_{\mathrm{A}}$ resultó buen indicador de intervención para las áreas de bajo impacto. En las áreas de mediano y alto impacto se analizó complementariamente la dominancia, la riqueza y la diversidad específica. Eremobelba zicsii, Oppiella nova y Epilohmannia pallida americana dominaron en suelos sanos. Se observó una reducción de la densidad de Hemileius initialis, Scheloribates curvialatus y Protoribates (Triangius) praeoccupatus conforme aumentó el grado de intervención antrópica. Se sugiere incluir la densidad de estas especies en un estimador de calidad de suelos urbanos. Tectocepheus velatus, Trachyoribates (Rostrozetes) ovulum y Acrotitia clavata, especies dominantes en sectores de alto impacto, fueron raras o estuvieron ausentes en los sectores protegidos del tránsito peatonal por lo que se proponen como resistentes a la intervención moderada.

Palabras clave: Oribatida, Bioindicadores, uso antrópico, suelos urbanos.

Accattoli, C. \& Salazar Martínez, A. 2012. Oribatid mites (Acari: Oribatida): anthropogenic impact indicators in urban forests from La Plata (Argentina). Acta Zoológica Mexicana (n. s.), 28(3): 550-565.

ABSTRACT. Seven green areas were sampled in the city of La Plata in order to test a human intervention index $\left(\mathrm{I}_{\mathrm{A}}\right)$ for urban soils. This index emerged from a research conducted in the Paseo del Bosque (La Plata, Argentina) and uses oribatid mites as bioindicators. $\mathrm{I}_{\mathrm{A}}$ was a good indicator of a human intervention in low impact areas. In high and medium impact areas species dominance, richness and diversity were analysed. Eremobelba zicsii, Opiella nova and Epilohmannia pallida americana were dominant in healthy soils. A density reduction of Hemileius initialis, Scheloribates curvialatus and Protoribates (Triangius) praeoccupatus was observed with and increasing degree of human intervention. We suggest that the density of these species should be included as an estimate of urban soils health. Tectocepheus velatus, Trachyoribates (Rostrozetes) ovulum and Acrotitia clavata were dominant in high impact areas

Recibido: 23/01/2012; aceptado: 20/08/2012. 
and rare or absent in healthy soils. This finding has led us to regard them as resistant to moderate intervention.

Key words: Oribatida, bioindicators, anthropogenic use, urban soils.

\section{INTRODUCCIÓN}

Cuando se aprobó el trazado de la ciudad de La Plata en 1882 se aplicaron criterios urbanísticos de vanguardia, reconociendo la necesidad de incluir espacios verdes en los ambientes urbanos (Olivier 2002). Fue así como se crearon parques, plazas y jardines en torno a los edificios públicos y en los extremos de sus ejes fundacionales se respetó la presencia de dos espacios verdes preexistentes. El actual Paseo del Bosque fue uno de ellos, está ubicado en el noreste de la ciudad, es el área verde de mayor superficie y es visitado regularmente por ciento de personas. Actualmente la ciudad cuenta con una macrotrama de espacios abiertos que le otorga $20 \mathrm{~m}^{2}$ de áreas verdes por habitante, superficie que duplica a la recomendada por la Organización Mundial de la Salud (Pesci 2003). Sin embargo la presión ambiental ejercida por los visitantes ha favorecido la erosión y compactación edáfica en parques y plazas (Accattoli 2010). En el Paseo del Bosque, los trabajos de mantenimiento involucran el retiro de materiales orgánicos, como el mantillo, disminuyendo la cantidad y cambiando la calidad del sustrato que inicia la red de detritos, lo que interfiere sobre la presencia de la fauna y el normal desarrollo del proceso de descomposición y consecuentemente sobre los procesos de pedogénesis.

En los últimos años se ha propuesto a la presencia y abundancia de microartrópodos para señalar la calidad del suelo (Iturrondobeitia et al. 2004; Parisi et al. 2005; Lavelle et al. 2006; Bedano 2007; Accattoli 2010). Los ácaros oribátidos (Acari: Oribatida) tienen gran potencial como bioindicadores ya que son uno de los grupos más abundantes de la mesofauna, tienen alta diversidad específica, pertenecen a un grupo trófico heterogéneo, poseen ciclos de vida relativamente largos ( 1 o 2 años) y tienen movilidad restringida por lo que no pueden escapar fácilmente de las situaciones de estrés ni aumentar su densidad poblacional en cortos períodos de tiempo (Linden et al. 1994; Behan-Pelletier 1999).

Entre los índices que se han desarrollado se hallan los que relacionan a la densidad de Oribtida con la de otros subórdenes de ácaros como Astigmata o Mesostigmata y Prostigmata (Oribátida+Mesostigmata)/ (Prostigamata+Astigmata). En ambos, los valores superiores a 1 permiten diferenciar ambientes con mayor calidad del suelo (Bedano 2007).

Aoki (1979) usó la proporción del número de individuos y el de las especies de oribátidos de distintos grupos como indicadores de intervención antrópica y propuso que la mayor abundancia o riqueza de Macropylina señala un menor grado de intervención antrópica. Dado que cambios en la abundancia relativa de las especies, riqueza específica, diversidad específica y composición específica pueden ser considerados 
como un aviso temprano de estrés (Gulvik 2007), estos parámetros se han empleado para evaluar cambios en su ambiente (Siepel 1996; Salazar \& Accattoli 2002; Salazar et al. 2006; Fredes et al. 2009; Accattoli 2010; Salazar et al. 2010). Las dificultades prácticas para implementar aspectos comunitarios son el escaso conocimiento de la fauna edáfica a nivel general y el esfuerzo que implica su determinación.

El empleo de oribátidos como indicadores de la calidad del suelo está en una etapa inicial y la mayoría de los índices se han aplicado en el estudio de agroecosistemas (Bedano 2007; Bedano et al. 2011).

Esta investigación da continuidad a trabajos previos (Accattoli et al. 2006; Accattoli et al. 2007; Salazar et al. 2007; Accattoli 2010; Salazar et al. 2010) y se basa en el análisis de la variación de las especies de oribátidos luego de la simulación de intervención antrópica.

Los objetivos de este trabajo son:

1. Elaborar un índice de calidad de suelo aplicable a suelos urbanos, basándose en la respuesta de la taxocenosis a los cambios impactos antrópicos.

Hipótesis 1: el tránsito peatonal genera cambios en el elenco oribatológico que sirven para la construcción de un índice de impacto antrópico aplicable a espacios verdes urbanos.

2. Poner a prueba dicho índice en áreas verdes urbanas diferenciadas por su superficie y ubicación respecto del centro de la ciudad.

Hipótesis 2: los valores del índice creado que señalen menor impacto antrópico se asociarán a parques y plazas de mayor extensión y más alejados del centro de la ciudad.

\section{MATERIALES Y MÉTODOS}

Las áreas verdes de estudio fueron: el Paseo del Bosque, los parques J. Vucetich, Saavedra, Castelli y Alberti; y las plazas España, San Martín y Olazábal ubicados en

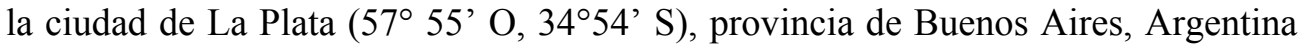
(Fig. 1). Los suelos dominantes en el área urbana de la ciudad pertenecen al orden Molisoles, subgrupo Argiudoles vérticos y están en gran parte modificados por acción antrópica, sobre todo en superficie (Hurtado et al. 2006). El trabajo fue realizado en dos etapas, en primera instancia se realizaron muestreos mensuales durante un ciclo anual en el Paseo del Bosque, con esos datos se elaboró un índice de intervención antrópica. Luego se procedió a muestrear parques y plazas con diferencias en su superficie y ubicación respecto del centro de la ciudad con el fin de poner a prueba dicho índice (Cuadro 1).

En investigaciones previas realizadas en el Paseo del Bosque se registraron valores de abundancia relativa, riqueza y diversidad específica de oribátidos, semejantes a los observados en ambientes nativos, por lo que se lo considera un sistema en buen esta- 


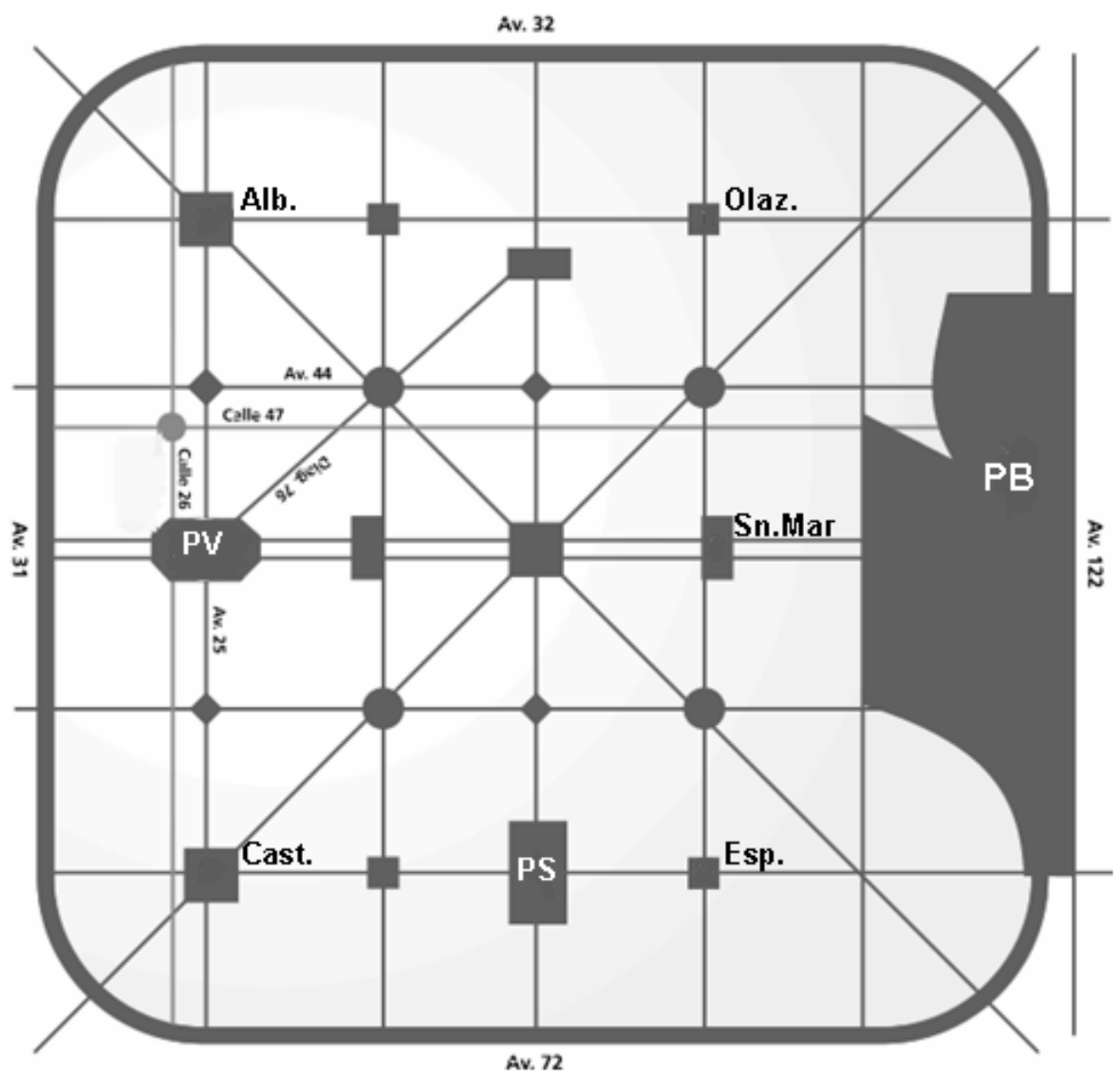

Figura 1. Plano de la ciudad de La Plata y ubicación de las áreas verdes urbanas muestreadas. Referencias: PB= Paseo del Bosque, PV: Parque Vucetich, PS: Parque Saavedra, Alb.: Parque Alberti, Cast.: Parque Castelli, Olaz.: Plaza Olazabal, Esp.: Plaza España, Sn.Mar.: Plaza San Martín.

do de conservación, que actuaría como reservorio de biodiversidad y fuente de fauna edáfica en la ciudad de La Plata (Salazar \& Rusiñol 2002; Salazar et al. 2010).

$1^{\text {a }}$ Etapa: En el Paseo del Bosque se tomaron muestras de suelo en un sector que ha permanecido aislado del tránsito peatonal durante 10 años en dos parcelas: testigo y experimental. En esta última se simuló el efecto del tránsito peatonal entre septiembre y noviembre de 2007 (Accattoli 2010). En 2008, se llevaron a cabo doce muestreos mensuales completando un ciclo anual. En cada uno de ellos se extrajeron al azar 8 muestras de suelo de $300 \mathrm{~cm}^{3}$ en ambas parcelas, 96 en total.

$2^{\text {a }}$ Etapa: En abril de 2010 se extrajeron 10 muestras de suelo al azar en los parques y plazas restantes. En los parques J. Vucetich y Saavedra se muestrearon tres secto- 


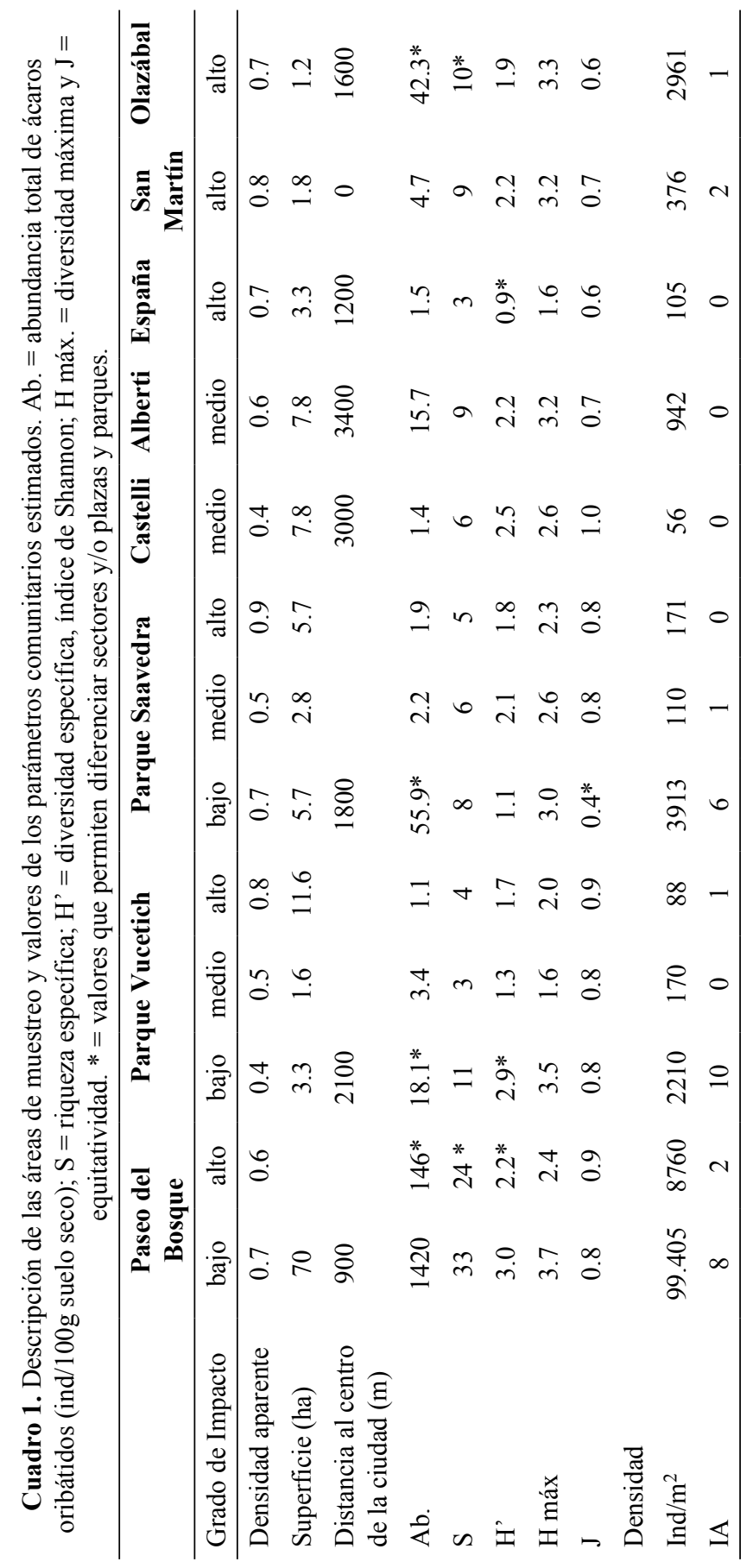


res con diferente intensidad de impacto antrópico (bajo, medio y alto), definiendo la intensidad en función del grado de deterioro observado visualmente (ausencia de cobertura herbácea y signos de erosión edáfica por tránsito peatonal); en las plazas, que constituyen sectores de tránsito frecuente, las muestras se retiraron desde lugares con $100 \%$ de cobertura vegetal y menor probabilidad de compactación por tránsito peatonal (Cuadro 1).

El material faunístico fue extraído en embudos Berlese de $2.5 \mathrm{~mm}$ de abertura de malla, durante quince días y conservado en alcohol 70\%. Los oribátidos fueron separados bajo microscopio estereoscópico e identificados a nivel especie (Balogh \& Balogh 1988, 1992a, 1992b; Martínez 2008; Subías 2010).

Para evaluar el grado de compactación del suelo se estimó la densidad aparente a través del método gravimétrico.

Análisis Ecológicos y Estadísticos. Se estimó la densidad de oribátidos expresándose como número de individuos por $\mathrm{m}^{2} \mathrm{y}$ la riqueza específica como número de especies. Para establecer la importancia de las especies de oribátidos se utilizaron porcentajes de abundancia relativa. Se consideraron especies dominantes aquellas cuyos valores de abundancia relativa fueron mayores a $3 \%$ y raras a aquellas cuya abundancia no superó el 1\%.

Se calculó la diversidad específica (H') a través del índice de Shannon, la diversidad máxima (H' máx.) y la equitatividad de Pielou (J) para cada una de las áreas estudiadas (Sáiz 1980).

La composición específica de la taxocenosis de oribátidos se comparó mediante el índice de Jaccard (Sáiz 1980) y los parámetros relativos a la densidad y a la diversidad específica con el test de Friedman $(\alpha=0.05)$. A través de un análisis de correspondencia canónica (CCA) se relacionó la distancia al centro de la ciudad, la superficie en hectáreas y la densidad aparente de los sitios con su composición específica.

Todos los test y análisis estadísticos fueron desarrollados empleando el software XLstat2010.excel, en su versión para Windows XP (Addinsoft 2010).

Al menos 10 ejemplares adultos de cada especie de oribátidos fueron depositados en la colección húmeda de la División Entomología del Museo de La Plata.

\section{RESULTADOS Y DISCUSIÓN}

1 atapa: Paseo del Bosque. Se recolectaron 5680 individuos pertenecientes a 40 especies de ácaros oribátidos (Cuadro 2). Entre los grupos más representativos por sus altos valores de abundancia relativa, riqueza específica y frecuencia se encontraron galúmnidos y ópidos. Ambos grupos evidenciaron cambios significativos que fueron asociados a la simulación del tránsito peatonal. Los galúmnidos presentan un amplio espectro trófico, son activos y capaces de recorrer mayores distancias que los ópidos, realizan migraciones verticales y se han asociado con suelos intervenidos. Los ópidos, en cambio, presentan mayor abundancia en suelos con menor intervención antrópica 
Cuadro 2. Lista de especies de ácaros oribátidos (Acari: Oribatida) detectadas en las áreas verdes de la ciudad de La Plata. $\bullet=$ especies halladas exclusivamente en el Paseo del Bosque; sin símbolo especies halladas en el Paseo del Bosque y en el resto de las plazas y parques de la ciudad.

\begin{tabular}{|c|c|c|}
\hline \multicolumn{3}{|c|}{ Acari: Oribatida } \\
\hline Subórdenes & Familias & Género / Especie \\
\hline Enarthronota & Hypoctoniidae Berlese, 1940 & $\begin{array}{l}\text { Eohypochthonius (Neoatrichosus) travei } \\
\text { Fernández, } 1984\end{array}$ \\
\hline Desmonomata & Camisiidae Oudemans, 1900 & Platynothrus robustior Berlese, $1916 \bullet$ \\
\hline \multirow[t]{5}{*}{ Mixonomata } & Euphthiracaridae Jaccot, 1930 & Acrotritia clavata clavata (Märkel, 1964) \\
\hline & Epilohmanniidae Oudemans, 1923 & $\begin{array}{l}\text { Epilohmannia pallida americana } \text { Balogh y } \\
\text { Mahunka, } 1981\end{array}$ \\
\hline & Lohmanniidae Berlese, 1916 & $\begin{array}{l}\text { Lohmannia (Lohmannia) juliae Mahunka, } \\
1984\end{array}$ \\
\hline & & $\begin{array}{l}\text { Torpacarus omttens omittens Grandjean, } \\
1950 \bullet\end{array}$ \\
\hline & & Papillacarus spinosus Alzuet, $1972 \bullet$ \\
\hline \multirow[t]{14}{*}{ Brachypylina } & Galumnidae Jaccot, 1925 & $\begin{array}{l}\text { Galumna (Galumna) pallida Hammer, } \\
1958\end{array}$ \\
\hline & & $\begin{array}{l}\text { Galumna (Galumna) flavellifera Hammer, } \\
1958\end{array}$ \\
\hline & & $\begin{array}{l}\text { Galumna (Galumna) reticulata Hammer, } \\
1958\end{array}$ \\
\hline & & $\begin{array}{l}\text { Galumna (Galumna) inexa Pérez-Iñigo y } \\
\text { Baggio, } 1986 \bullet\end{array}$ \\
\hline & & Carinogalumna clericata Berlese, 1914 \\
\hline & Scheloribatidae & Scheloribates curvialatus Hammer, 1961 \\
\hline & Balogh y Balogh, 1984 & $\begin{array}{l}\text { Hemileius (Hemileius) laticlava Hammer, } \\
1961\end{array}$ \\
\hline & & $\begin{array}{l}\text { Hemileius (Hemileius) initialis Berlese, } \\
1908\end{array}$ \\
\hline & & $\begin{array}{l}\text { Hemileius (Hemileius) suramericanus } \\
\text { Hammer, } 1958\end{array}$ \\
\hline & & $\begin{array}{l}\text { Hemileius (Hemileius) trichosus Hammer, } \\
1958\end{array}$ \\
\hline & Oribatellidae Jaccot, 1925 & $\begin{array}{l}\text { Oribatella (Oribatella) neonominata } \\
\text { Hammer, } 1977 \bullet\end{array}$ \\
\hline & Ceratozetidae Jaccot, 1925 & Sphaerozetes (Porozetes) sp Hammer, 1962 \\
\hline & Mochlozetidae Grandjean, 1960 & Mochlozetes sp Grandjean, $1930 \bullet$ \\
\hline & Xylobatidae Balogh y Balogh, 1984 & $\begin{array}{l}\text { Protoribates (Triangius) praeoccupatus } \\
\text { nom. nov Hammer, } 1972\end{array}$ \\
\hline
\end{tabular}


Cuadro 2. Continuación.

\section{Acari: Oribatida}

\begin{tabular}{|c|c|c|}
\hline Subórdenes & Familias & Género / Especie \\
\hline & & Perxylobates sp Hammer, 1972 \\
\hline & Microzetidae Grandjean; 1936 & $\begin{array}{l}\text { Berlesezetes brasilozetoides Balogh y } \\
\text { Mahunka, } 1981\end{array}$ \\
\hline & \multirow[t]{2}{*}{ Plateremaeidae Trägard, 1931} & $\begin{array}{l}\text { Plateremaeus costulatus Balogh y } \\
\text { Mahunka, } 1978 \bullet\end{array}$ \\
\hline & & Pheroliodes minutus Baranek, $1984 \bullet$ \\
\hline & Eremobelbidae Balogh, 1961 & $\begin{array}{l}\text { Eremobelba zicsii Balogh y Mahunka, } \\
1969 \bullet\end{array}$ \\
\hline & Protoribatidae Balogh y Balogh, 1984 & Totobates discifer Hammer, 1961 \\
\hline & Tectocepheidae Grandjean, 1958 & Tectocepheus velatus Michael, 1980 \\
\hline & Haplozetidae Grandjean, 1936 & $\begin{array}{l}\text { Trachyoribates (Rostrozetes) ovulum } \\
\text { ovulum Berlese } 1908\end{array}$ \\
\hline & Oripodidae Jaccot, 1925 & Oripoda maxensis Mahunka, $1984 \bullet$ \\
\hline & Oribatulidae Thor, 1929 & $\begin{array}{l}\text { Dometorina chilensis (Hammer, 1962) } \\
\text { (Cryptozetes) • }\end{array}$ \\
\hline & Suctobelbidae Grandjean, 1954 & $\begin{array}{l}\text { Suctobelbella (Flagrosuctobelba) } \\
\text { multiplumosa (Hammer, 1969) }\end{array}$ \\
\hline & \multirow[t]{8}{*}{ Oppiidae Grandjean, 1954} & Oppiella nova Oudemans, $1902 \bullet$ \\
\hline & & $\begin{array}{l}\text { Membranoppia (Membranoppia) } \\
\text { breviclava (Hammer, 1958) • }\end{array}$ \\
\hline & & $\begin{array}{l}\text { Lanceoppia (Lancelalmoppia) nodosa } \\
\text { (Hammer, 1958) }\end{array}$ \\
\hline & & $\begin{array}{l}\text { Striatoppia tribuliformi Balogh y } \\
\text { Mahunka,1981 }\end{array}$ \\
\hline & & Ramusella (Inscultoppia) sp $1 \bullet$ \\
\hline & & Ramusella (Inscultoppia) sp 2 \\
\hline & & Ramusella (Ramusella) sp $1 \bullet$ \\
\hline & & Ramusella (Ramusella) sp $2 \bullet$ \\
\hline
\end{tabular}

donde se favorece el desarrollo de mantillo y la presencia de comunidades fúngicas, que constituyen su principal fuente de alimento (Guevara et al. 2002; Woas 2002). Entre las especies identificadas en el Paseo del Bosque, 5 pertenecieron a Galumnidae y 8 a Oppiidae. Al comparar el porcentaje de abundancia relativa promedio anual de estas dos familias, entre parcelas testigo e intervenida, los valores fueron $69 \%$ para 
ópidos y $17 \%$ para galúmnidos en la primera y $16 \%$ y $45 \%$, respectivamente, en la segunda. Estos resultados coinciden con las observaciones realizadas por Guevara et al. (2002) y Woas (2002).

A partir de los resultados obtenidos y de los antecedentes expuestos se propuso utilizar la siguiente proporción entre la abundancia de Oppiidae y Galumnidae como indicadora de intervención antrópica:

$$
\mathrm{I}_{\mathrm{A}}=\frac{\text { (abundancia de ópidos }+ \text { abundancia de galúmnidos) }}{\text { abundancia de galúmnidos }}
$$

Un valor de $\mathrm{I}_{\mathrm{A}}$ igual a 1 indica ausencia de ópidos y sitios con mayor grado de intervención antrópica, el valor 2 indica igual abundancia de ópidos y galúmnidos. Los valores mayores que 2 indican predominio de los ópidos sobre los galúmnidos y sitios donde los procesos y funciones no se encuentran limitados por factores culturales. Para facilitar su interpretación se consideraron sólo los valores enteros del índice.

Al calcular $\mathrm{I}_{\mathrm{A}}$ con los valores de abundancia promedio anual de ópidos y galúmnidos para las parcelas testigo e intervenida del Paseo del Bosque, el índice presentó un valor igual a 8 en la primera e igual a 2 en la segunda (Cuadro 1). La variación anual de $I_{A}$ en la parcela intervenida fue baja y se registraron valores menores que 2 durante la mayor parte del período de muestreo. Transcurrido el año se observó una tendencia hacia valores mayores, lo que se interpretó como signo de recuperación del sistema. Estos resultados corroboraron la sensibilidad del índice en el Paseo del Bosque y para su interpretación se propuso como valor de referencia 4, valor que correspondió a la moda estimada en la parcela testigo durante el ciclo anual.

$2^{\mathbf{a}}$ Etapa: Parques y Plazas. Se recolectaron 1,117 individuos de oribátidos, comprendidos en 17 especies (Cuadro 2). Los valores de $\mathrm{I}_{\mathrm{A}}$ registrados en los sectores de bajo impacto de los parques Vucetich y Saavedra fueron 10 y 6 respectivamente; en la plaza San Martín, considerada con alto grado de impacto antrópico, $\mathrm{I}_{\mathrm{A}}$ fue igual a 2 (Cuadro 1). En el resto de los sectores (medio y alto impacto) $\mathrm{I}_{\mathrm{A}}$ fue igual a 1 o no fue posible estimarlo por ausencia de ambas familias. Estos resultados corroboraron parcialmente la utilidad del índice como indicador de impacto antrópico, en consecuencia se analizó la similitud taxonómica y la dominancia de especies en las áreas de estudio buscando elementos complementarios de evaluación de impacto.

A través del análisis de similitud taxonómica $(\mathrm{Sj}=$ índice de Jaccard (Fig. 2) se reúnen dos grupos de sitios: el primero, estuvo formado por las parcelas del Paseo del Bosque y el segundo, conformado por el resto de los parques y plazas analizados. Este último presentó una alta heterogeneidad interna, siendo el elenco oribatológico de plaza España el más disímil.

El análisis de parámetros comunitarios, como el esquema de dominancia específica y la diversidad, separó al Paseo del Bosque como el área verde con mayor 


\section{Dendrograma}

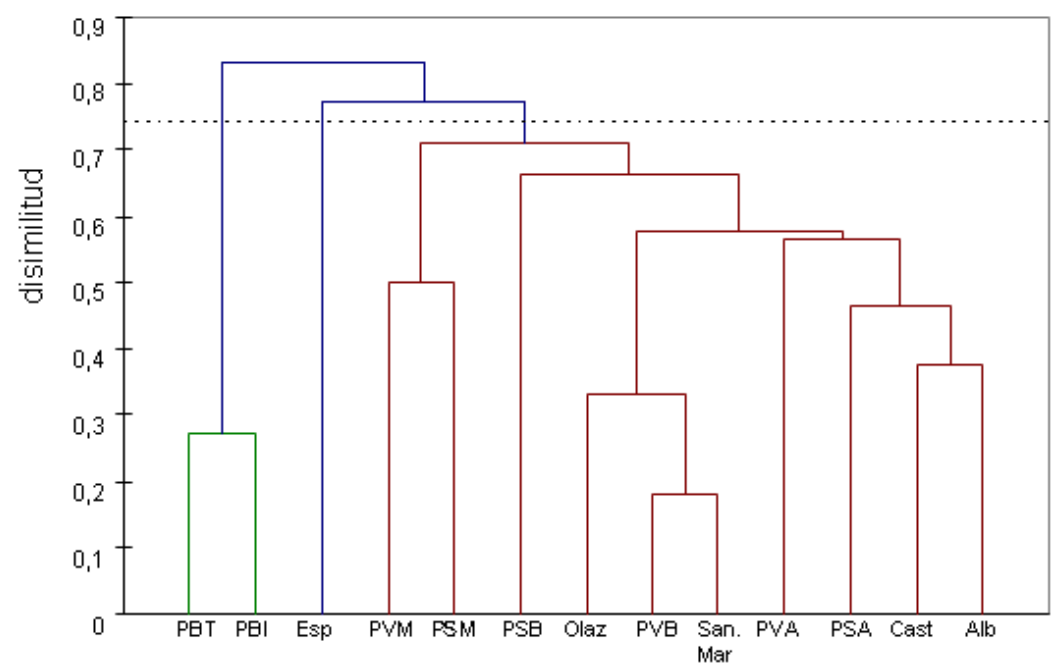

Figura 2. Dendrograma de Disimilitud ( $\mathrm{D}=1$ - Índice de Jaccard). $\mathrm{PB}=\mathrm{Paseo}$ del Bosque, $\mathrm{PBT}=$ parcela testigo, $\mathrm{PBI}=$ parcela intervenida; $\mathrm{PV}$ : Parque Vucetich, $\mathrm{PVB}=$ bajo impacto, $\mathrm{PVM}$ : medio impacto, PVA: alto impacto; PS: Parque Saavedra, PSB=bajo impacto, PSM= medio impacto, PSA=alto impacto; Alb.: Parque Alberti, Cast.: Parque Castelli, Olaz.: Plaza Olazabal, Esp.: Plaza España, Sn.Mar.: Plaza San Martín.

abundancia y diversidad específica entre las estudiadas (Cuadro 1), con especies dominantes propias de la parcela testigo como Eremobelba zicsii, Oppiella nova y Epilohmannia pallida americana. Las últimas fueron detectadas en entisoles de sitios con uso recreativo en Miramar (Mar del Plata, Argentina), asociadas a sectores con bajo grado de uso antrópico, con mayor heterogeneidad espacial y mayor disponibilidad de recursos tróficos (Fredes et al. 2009). Estos resultados sugieren que su presencia refleja menor actividad antrópica.

La cantidad de oribátidos recolectada en los sectores con bajo impacto antrópico de los parques Saavedra y Vucetich fue significativamente mayor que en el resto. Este sector, en el parque Saavedra concentró 93\% de los oribátidos, 8 especies y baja diversidad específica atribuible a la predominancia de Hemileius initialis. En el parque Vucetich reunió $80 \%$ del total recolectado y presentó una riqueza específica significativamente mayor que el resto de sus sectores (Fig. 3; Cuadro 1).

Hemileius initialis, Scheloribates curvialatus y Protoribates (Triangius) praeoccupatus dominaron numéricamente en los sectores con bajo impacto de los parques y en el bosque en general y su abundancia relativa fue notablemente menor en el resto de los sectores estudiados, observándose una reducción conforme aumentó el nivel de 
a-
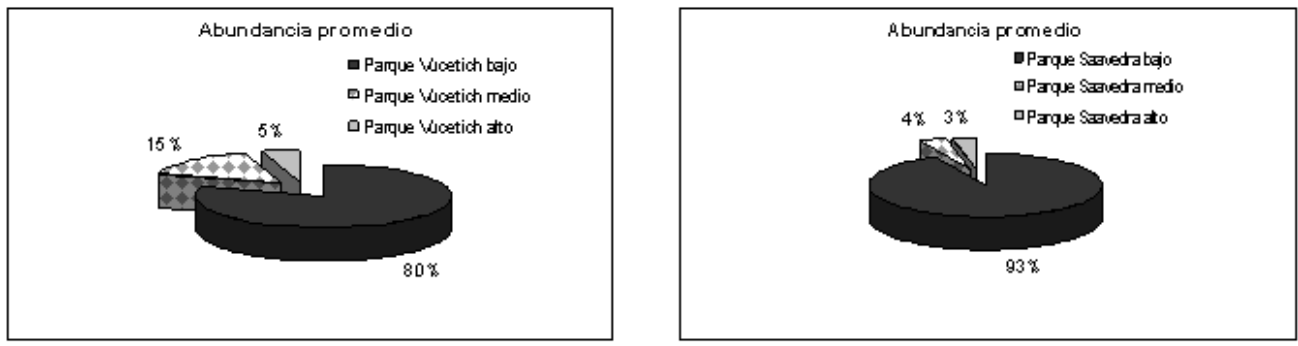

b-
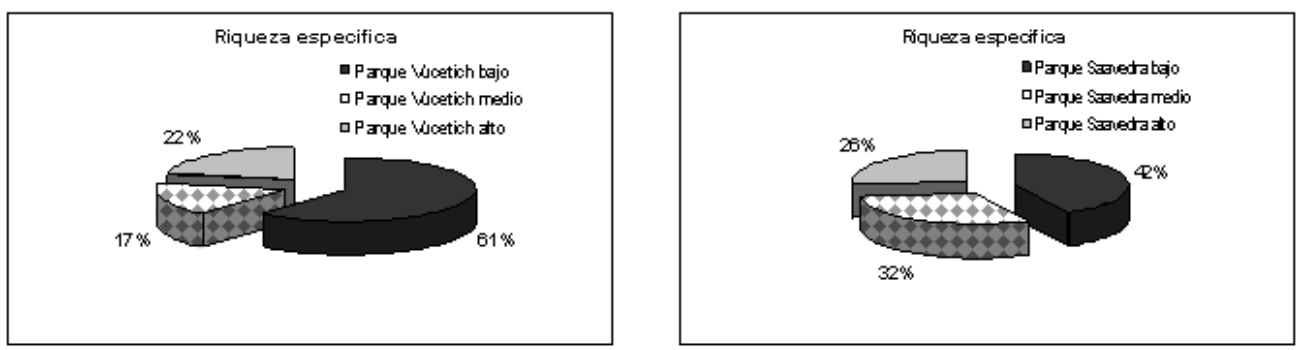

c-
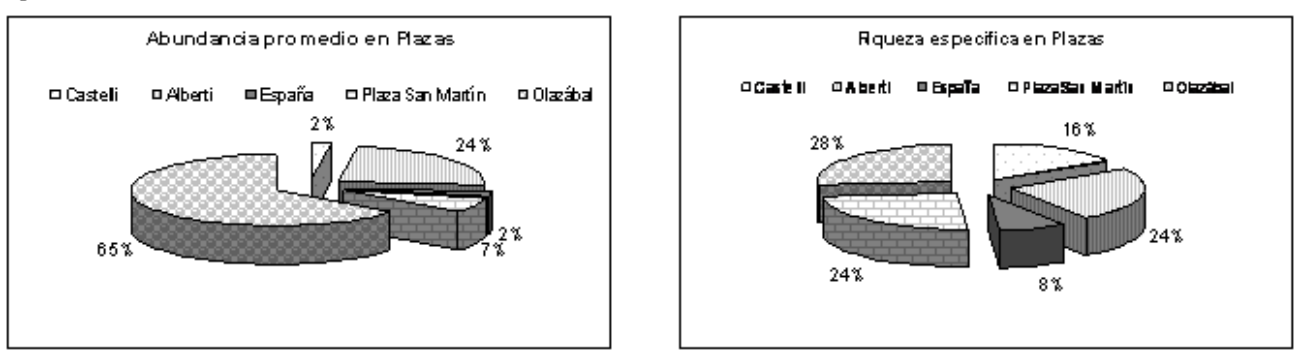

Figura 3. a- Porcentajes de abundancia promedio de oribátidos recolectados en los Parques Vucetich y Saavedra; b- Porcentajes de riqueza específica de oribátidos recolectados en los dichos parques. cPorcentajes de abundancia promedio y de riqueza específica de oribátidos recolectados en los parques y plazas de menor superficie.

intervención antrópica. Varios autores han señalado que estas especies poseen escasa selectividad trófica y resultan comunes a suelos de bosques nativos y urbanos (Wallwork 1983; Fernández et al. 1991; Jung et al. 1998; Salazar et al. 2010). Asociamos su permanencia en los sectores de tránsito peatonal intenso a su condición de generalistas. Consideramos que la densidad de estas especies puede ser útil para estimar el grado de intervención antrópica. 
Plaza España se diferenció del resto de las áreas verdes estudiadas por presentar los menores valores de densidad y diversidad de oribátidos, con Acrotritia clavata clavata como especie dominante (Fig. 3; Cuadro 1). Es posible que A. clavata con quelíceros cortos, gruesos y altamente esclerotizados se asocie con los restos recalcitrantes del mantillo proveniente de Tilia moltkei (tilo), que en la época de estudio cubrían el suelo, ya que muchas especies de Euphthiracaridae con estas características han sido clasificadas como xilófagas.

Sólo se observaron diferencias estadísticamente significativas entre la densidad de oribátidos de plaza España y plaza Olazábal (Fig. 3; Cuadro 1). Esta última, presentó valores de abundancia y riqueza específica semejantes a los observados en los sectores de bajo impacto de los parques y la misma especie dominante, Hemileius initialis. Al ser un área verde de escasa superficie y muy próxima al centro de la ciudad, se esperaba que se diferenciara de sectores con bajo grado de impacto antrópico, sin embargo los resultados obtenidos son opuestos a nuestras expectativas. Una posible explicación es que su superficie y su proximidad a una de las vías de salida de la ciudad, con gran afluencia de tránsito vehicular, hacen de esta plaza un sector poco propicio para la recreación y por consiguiente la intensidad del tránsito peatonal sería menor.

En aquellas plazas de menor superficie y sectores de parques con evidentes signos de impacto antrópico, se registraron Tectocepheus velatus, Trachyoribates (Rostrozetes) ovulum y Acrotritia clavata clavata como especies dominantes y/o con altos valores de abundancia relativa. Las mismas en el Paseo del Bosque y en los sectores de bajo impacto fueron especies raras o no fueron registradas. Estos resultados permiten proponerlas como especies resistentes a la intervención antrópica moderada. Su presencia y/o abundancia podrían emplearse como indicadoras de impacto ambiental.

El análisis de correspondencia canónica (CCA) ordenó a las especies de oribátidos en dos ejes que representan gradientes de cambio de algunas de las variables consideradas. Las áreas más próximas unas de otras en el espacio son semejantes en composición específica. El primer eje ( $\mathrm{F} 1=86,20$ se asoció con la superficie de las áreas muestreadas y el segundo con la densidad aparente del suelo $(\mathrm{F} 2=11,03)$ (Fig. 4a). Las parcelas del Paseo del Bosque, consideradas las áreas de la ciudad con suelos de mayor calidad (Accattoli 2010), se asociaron por su mayor extensión y se diferenciaron entre si por los valores de densidad aparente del suelo. Las áreas verdes con alto grado de impacto antrópico, conformaron un segundo grupo en el cuadrante superior izquierdo, reunidas por la presencia de Tectocepheus velatus y Acrotritia clavata clavata como especies características, asociadas a sectores de superficie escasa y alta densidad aparente (Fig. 4b). Un tercer grupo incluyó a los lugares de menor superficie y valores intermedios de densidad aparente.

Los espacios con menor grado de impacto antrópico, se agruparon en el cuadrante inferior izquierdo en un cuarto grupo, asociados a valores intermedios de superficie, 
a-

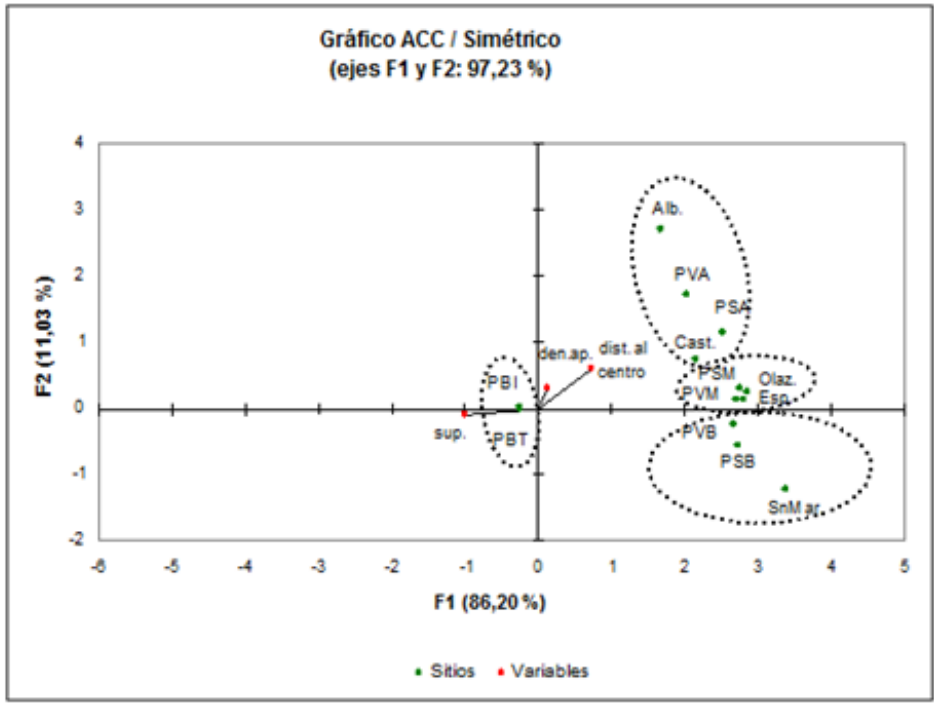

b-

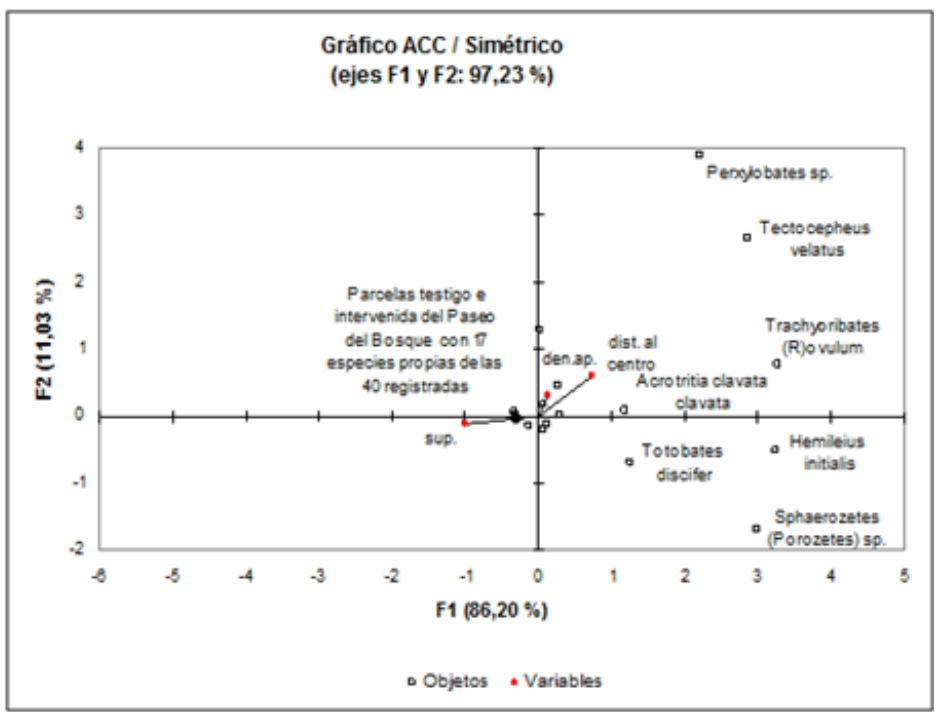

Figura 4. Análisis de Correspondencia Canónica (CCA). Referencias: sup.: superficie en hectáreas, den.ap.: densidad aparente del suelo, dis. al centro: distancia al centro en metros. $\mathrm{PB}=\mathrm{Paseo}$ del Bosque, $\mathrm{PBT}=$ parcela testigo, $\mathrm{PBI}=$ parcela intervenida; $\mathrm{PV}$ : Parque Vucetich, $\mathrm{PVB}=$ bajo impacto, PVM: medio impacto, PVA: alto impacto; PS: Parque Saavedra, PSB=bajo impacto, PSM= medio impacto, PSA=alto impacto; Alb.: Parque Alberti, Cast.: Parque Castelli, Olaz.: Plaza Olazabal, Esp.: Plaza España, Sn.Mar.: Plaza San Martín. 
bajos valores de densidad aparente y unidos por la dominancia de Hemileius initialis. Se destaca la presencia de plaza San Martín, considerada a priori como sector de alto impacto antrópico debido a su ubicación céntrica y a que cientos de individuos la transitan diariamente. Su pertenencia a este grupo de sectores nos permite sugerir que la salud edáfica también está influenciada por el diseño del parque. En plaza San Martín existen senderos establecidos que invitan a las personas a caminar sin entrar en contacto con el césped, esto permitiría que la taxocenosis de oribátidos muestre una composición específica similar a la de áreas verdes urbanas más extensas y menos concurridas.

En cuanto a la riqueza específica se observó un gradiente en el eje 1, de izquierda a derecha, que permite diferenciar al Paseo del Bosque del resto de las áreas verdes por presentar el mayor número de especies.

\section{CONCLUSIONES}

El índice $\mathrm{I}_{\mathrm{A}}$ propuesto resultó buen indicador de intervención antrópica, útil para diagnósticos iniciales. Respecto a otros índices utilizados (Aoki 1979; Parisi et al. 2005; Bedano et al. 2007) presenta ventajas de índole metodológica porque las observaciones se realizan sobre dos familias de oribátidos que son abundantes en sistemas urbanos (Galumnidae y Oppiidae), y son reconocibles bajo microscopio estereoscópico lo que simplifica su identificación y conteo.

En sitios afectados por el uso antrópico intensivo ópidos y/o galúmnidos estuvieron ausentes por lo que se analizaron aspectos complementarios para evaluar la intervención antrópica. La dominancia de las especies se asoció al grado de uso de los sectores públicos por lo que resulta un aspecto a considerar en una propuesta futura. Tectocepheus velatus, Trachyoribates (Rostrozetes) ovulum y Acrotritia clavata cla$v a t a$, especies con altos valores de abundancia relativa en los sectores de alto impacto fueron raras o estuvieron ausentes en suelos más escaso o nulo uso antrópico. Estos resultados permiten proponerlas como especies resistentes a la intervención antrópica moderada. Su presencia y/o abundancia podría combinarse en la confección de un nuevo indicador de impacto ambiental. La menor abundancia de Eremobelba zicsii, Oppiella nova, Epilohmannia pallida americana en los sitios con suelos más compactados permite proponerlas como especies sensibles a la intervención antrópica.

Las áreas verdes urbanas presentan un mosaico de situaciones espaciales resultante de la intensidad de uso antrópico. Actualmente, las plazas y parques comparados, reciben los mismos procedimientos de cuidado. Se detectaron diferencias entre ellos asociadas a la intensidad del tránsito peatonal, y de acuerdo a nuestros resultados la superficie y el diseño de parques y plazas deben ser considerados al establecer pautas de manejo y cuidado del suelo en las ciudades. Conservar sus suelos con bajo grado de impacto antrópico es necesario para facilitar su permanencia, además de favorecer el adecuado manejo del sistema con un bajo aporte de insumos. 
Agradecimientos. Las autoras agradecen a la Lic. Verónica Bernaba Laborde por la lectura crítica del manuscrito.

\section{LITERATURA CITADA}

Addinsoft. 2010. XLSTAT 2010. Data analysis and statistical software for Microsoft Excel, Paris, France.

Accattoli, C., Morelli, I.\& Salazar, A. 2006. Efecto de diferentes grados de intervención antrópica sobre la microflora y la mesofauna del suelo. Comunicación en congreso: XX Congreso Argentino de Ciencias del Suelo. Salta y Jujuy, Argentina. Resúmenes, pg: 205. Resumen extendido: versión digital.

Accattoli, C., Salazar Martínez, A., Rusiñol, P. \& Schnack, J. A. 2007. Oribátidos de un parque urbano de la ciudad de La Plata, Buenos Aires, Argentina. Revista de la Sociedad Entomológica Argentina, 64: 175.

Accattoli, C. 2010. Efectos de la perturbación antrópica en la estructura y dinámica de la taxocenosis de oribátidos (Acari: Oribatida) y de la comunidad herbácea del Paseo del Bosque (La Plata, provincia de Buenos Aires). Trabajo de tesis doctoral. Facultad de Ciencias Naturales y Museo. UNLP. $192 \mathrm{pp}$.

Aoki, J. I. 1979. Difference in sensitivities of oribatid families to environmental change by human impacts. Reveu DÉcologie et de Biologie du Sol, 16: 415-422.

Balogh, J. \& Balogh, P. 1988. Oribatid mites of the neotropical region I. In: Balogh, J. \& S. Mahunka (Eds). The soil of the world. Akad. Kiadó, Budapest. Vol.II. 335 pp.

Balogh, J. \& Balogh, P. 1992 a. The Oribatid Mites of the World. Volume I. Hungarian Natural History Museum. Budapest. 262 pp.

Balogh, J. \& Balogh, P. 1992 b. The Oribatid Mites of the World. Volume II. Hungarian Natural History Museum. Budapest. 375 pp.

Bedano, J. C. 2007. El rol de la mesofauna edáfica en la evaluación de la calidad del suelo. In: Thuar, A., F. Cassán y C. Olmedo. De la biología del suelo a la agricultura. Universidad Nacional de Río Cuarto, Argentina. $291 \mathrm{pp}$.

Bedano, J.C., Domínguez, A. \& Arolfo, R. 2011. Assessment of soil biological degradation using mesofauna. Soil \& Tillage Research, 117: 55-60.

Behan-Pelletier, V. M. 1999. Oribatid mite biodiversity in agroecosystems: role for bioindication. Agriculture, Ecosystems and Environment, 74: 411- 423.

Fernández N., Velis, G. \& Martínez, P. A. 1991. Prelarvae of oribatids mites. Redia, 74: 343-353.

Fredes, N. A., Martínez, P. A., Bernava Laborde, V. \& Osterrieth, M. L. 2009. Microartrópodos como indicadores de disturbio antrópico en entisoles del área recreativa de Miramar, Argentina. Ciencia del Suelo, 27: 89-101.

Guevara R., Villedo, L. \& Najera, A. 2002. Soil mesofauna patterns and experiments on leaf-litter mite fungivory: preferentes, effects on fungal reproduction and decomposition. Acta Zoológica Mexicana, (n. s.), 87: 1 -15.

Gulvik, M. E. 2007. Mites (Acari) as Indicators of Soil Biodiversity and Land Use Monitoring: a review. Polish Journal of Ecology, 55: 415-440.

Hurtado, M. A., Giménez, J. E. \& Cabral, M. G. 2006. Análisis ambiental del partido de La Plata. Aportes al ordenamiento territorial. Instituto de Geomorfología y Suelos. Consejo Federal de Inversiones. $1^{\text {a }}$. Edición. $123 \mathrm{pp}$.

Iturrondobeitia, J. C., Caballero, A. I. \& Arroyo, J. 2004. Avances en el uso de los Ácaros Oribátidos como indicadores de las condiciones edáficas. MUNIBE (Suplemento/Gehigarria), 21: 70-91. 
Jung, C. E., Jonn-Ho Lee, Yun-Hwan Bae \& Seong-Sik Choi. 1998. Diversity of Oribatid Mites (Acari: Oribatida) in Namsan and Kwangreung Deciduous Forests. Korean J. Soil Zoology, 3: 91105.

Lavelle, P., Decaëns, T., Aubert, M., Barot, S., Blouin, M., Bureau, F., Margerie, P., Mora, P.\& Rossi, J. P. 2006. Soil invertebrates and ecosystem services. European Journal of Soil Biology, 42: S3-S15.

Linden, D., Hendrix, F., Coleman, D. \& Van Vliet, P. 1994. Fauna indicators of Soil Quality. In: Doran, J., Coleman, D., Bezdicek, D. \& Stewart, B. (Eds.) Soil Science Society of America - American Society of Agronomy, 35: 91-106.

Martínez, P. A. 2008. Oribatida, pp.129-140. In: Biodiversidad de artrópodos argentinos. Sociedad Entomológica Argentina ediciones, 2: 615 pp.

Olivier, S. 2002. El Bosque en mi ciudad. Congreso: El Patrimonio Urbano del Siglo XIX. Urbanismo, Arquitectura, Ambiente y Sociedad Republicana. Desarrollo Sustentable en Tiempos de Crisis. Comunicación. pp: 19-27.

Parisi, V., Menta, C., Gardi, C., Jacomini, C. \& Mozzanica, E. 2005. Microartropod communities as a tool to assess doil quality and biodiversity: a new approach in Italy. Agriculture, Ecosystems and Environment, 105: 323-333.

Pesci, R. 2003. La Plata, Ciudad Patrimonio. Edición: COMUNIC/AM, Fundación CEPA. Argentina. $144 \mathrm{pp}$.

Sáiz, F. 1980. Experiencias en el uso de criterios de similitud en el estudio de comunidades. Archivos de Biología y Medicina Experimentales, 13: 387-402.

Salazar Martínez, A. \& Accattoli, C. 2002. Composición de la taxocenosis de oribátidos (Acari: Oribatida) de un pastizal de la provincia de Buenos Aires. Neotropica, 48: 73-75.

Salazar Martínez, A. \& Rusiñol, P. 2002. Mesofauna del Paseo del Bosque, La Plata, Argentina. Neotropica, 48: 51-57.

Salazar, A., Accattoli, C., Martínez, P. A. \& Schnack, J. A. 2006. Nuevas citas de ácaros oribátidos (Acari: Oribatida) para la Argentina. Revista de la Sociedad Entomológica Argentina, 65: 19-22.

Salazar Martínez A., Accattoli, C. \& Schnack, J. A. 2007. Oribátidos arborícolas del "Paseo del Bosque" (La Plata, provincia de Buenos Aires, Argentina). Revista de la Sociedad Entomológica Argentina, 66: 159-163.

Salazar Martínez, A., Accattoli, C., Martínez, P. \& Schnack, J. A. 2010. Diversidad de oribátidos (Acari: Oribatida) del "Paseo del Bosque", La Plata (Buenos Aires, Argentina). Ciencia del Suelo, 28: 181-190.

Siepel, H. 1996. Biodiversity of soil microarthropods: the filtering of species. Biodiversity and Conservation, 5: 251-260.

Subías, L. S. 2010. Actualización on-line. Listado sistemático, sinonímico y biogeográfíco de los Ácaros Oribátidos (Acariformes, Oribatida) del mundo (1758-2002). Graellsia, 60 (número extraordinario): $305 \mathrm{pp}$.

Wallwork, J. A. 1983. Oribatids in Forest Ecosystems. Annual Review of Entomology, 28: 109-130.

Woas, S. 2002. Morphological organization and systematic groups of Oribatida. Joachim Adis (Ed.). Amazonian Arachnida and Myriapoda, 21-291. 